\section{BRAZIULIAN JOURNAL \\ OF MEDICAL AND BIOLOGICAL RESEARCH}

www.bjournal.com.br
ISSN 1414-431X

Volume 45 (12) 1102-1340 December 2012

\section{BIOMIDICAL SCIENCES}

AND

CLINICAL INVESTIGATION

Braz J Med Biol Res, December 2012, Volume 45(12) 1234-1239

doi: 10.1590/S0100-879X2012007500158

Depressed nNOS expression during spine transition in the developing hippocampus of FMR1 KO mice

Qin Xu, Zhiwei Zhu, Jialu Xu, Weizhong Gu and Zhengyan Zhao

The Brazilian Journal of Medical and Biological Research is partially financed by

\section{욛NPq}

Ministério

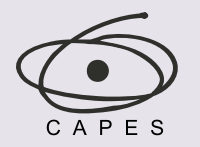

Ministério da Educação

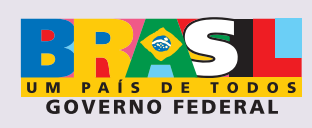

DTFAPESP

Institutional Sponsors

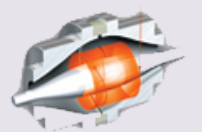

\section{SHIMADZU} UNICAMP

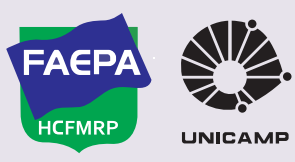




\title{
Depressed nNOS expression during spine transition in the developing hippocampus of FMR1 KO mice
}

\author{
Qin Xu1 ${ }^{1}$, Zhiwei Zhu ${ }^{1}$, Jialu Xu1 ${ }^{1}$, Weizhong $\mathrm{Gu}^{2}$ and Zhengyan Zhao ${ }^{1}$ \\ ${ }^{1}$ Department of Children's Health Care, Children's Hospital, Zhejiang University, Hangzhou, Zhejiang, China \\ 2Department of Pathology, Children's Hospital, Zhejiang University, Hangzhou, Zhejiang, China
}

\begin{abstract}
Nitric oxide (NO), synthesized as needed by NO synthase (NOS), is involved in spinogenesis and synaptogenesis. Immature spine morphology is characteristic of fragile $X$ syndrome (FXS). The objective of this research was to investigate and compare changes of postnatal neuronal NOS (nNOS) expression in the hippocampus of male fragile X mental retardation 1 gene knockout mice (FMR1 KO mice, the animal model of FXS) and male wild-type mice (WT) at postnatal day 7 (P7), P14, P21, and P28. nNOS mRNA levels were analyzed by real-time quantitative PCR $(N=4-7)$ and nNOS protein was estimated by Western blot $(\mathrm{N}=3)$ and immunohistochemistry $(\mathrm{N}=1)$. In the PCR assessment, primers 5'-GTGGCCATCGTGTCCTACCATAC-3' and 5'-GTTTCGAGGCAGGTGGAAGCTA-3' were used for the detection of nNOS and primers 5'-CCGTTTCTCCTGGCTCAGTTTA-3' and 5'-CCCCAATACCACATCATCCAT-3' were used for the detection of $\beta$-actin. Compared to the WT group, nNOS mRNA expression was significantly decreased in FMR1 KO mice at P21 (KO: $0.2857 \pm 0.0150$, WT: $0.5646 \pm 0.0657$; P < 0.05). Consistently, nNOS immunoreactivity also revealed reduced staining intensity at P21 in the FMR1 KO group. Western blot analysis validated the immunostaining results by demonstrating a significant reduction in nNOS protein levels in the FMR1 KO group compared to the WT group at P21 (KO: $0.3015 \pm 0.0897, \mathrm{WT}: 1.7542 \pm 0.5455 ; \mathrm{P}<0.05)$. These results suggest that nNOS was involved in the postnatal development of the hippocampus in FXS and impaired NO production may retard spine maturation in FXS.
\end{abstract}

Key words: FXS; nNOS; NO; Dendritic spine; Hippocampus

\section{Introduction}

Fragile $\mathrm{X}$ syndrome (FXS) is one of the most prevalent inherited mental disorders. It is caused by dynamic expansion of CGG repeats in the fragile $X$ mental retardation 1 (FMR1) gene, leading to transcriptional silencing of fragile mental retardation protein (FMRP) (1). FMRP interferes with gene expression at the RNA level (2). Subjects with FXS and FMR1 knockout (KO) mice have been shown to exhibit immature spine characteristic including elevated density and length of dendritic spines in the brain, which may underlie the cognitive and behavioral abnormalities in FXS $(3,4)$.

During spine morphogenesis, failure to establish normal connections with presynaptic axon terminals may, to some extent, contribute to an immature appearing profile of spine morphologies $(5,6)$. Changes in synaptic connections are considered essential for learning and memory formation (7). Nitric oxide (NO), as a neurotransmitter, is synthesized as needed by $\mathrm{NO}$ synthase (NOS) from its precursor L-arginine.
Prior studies have shown that $\mathrm{NO}$ is involved in spinogenesis and synaptogenesis (8-10). It has also been shown that NO can stimulate axons located around the spines to differentiate into varicosities to form multi-innervated spines (11). Based on the reported role for $\mathrm{NO}$ in spinogenesis and synaptogenesis and the observed immature spine morphology characteristic of FXS, we hypothesized that altered NO formation may be involved in spine malformation in FXS. Using an animal model of FXS, FMR1 KO mice, we compared the expression of neuronal NOS (nNOS) mRNA and protein levels from the hippocampi of wild-type (WT) and FMR1 KO mice at four time points after birth.

\section{Material and Methods}

\section{Animals}

FVB inbred strain mice, including FMR1 KO (FVB.129P2-

Correspondence: Zhengyan Zhao, Department of Children's Health Care, Children's Hospital, Zhejiang University, 57 Zhugan Xiang, Hangzhou 310003, Zhejiang, China. Fax: +86-571-8703-3296. E-mail: zhaozy@zju.edu.cn

Received November 19, 2011. Accepted April 3, 2012. Available online October 5, 2012. Published December 17, 2012. 
Fmr1tm1Cgr/J) and WT mice (FVB.129P2-Pde6b+ Tyrc-ch/ AntJ), were purchased from the Jackson Laboratory (USA). Animals were housed in a room with a 12-h light/dark cycle with access to food and water ad libitum. Each male was housed with a female in a plastic cage. $F_{1}$ pups were weaned and separated according to gender at 28 days. Each male $F_{1}$ mouse was mated with one female when they were 3 months old. At postnatal day 7 (P7), P14, P21, and P28 (day of birth was $P 0$ ), 4 to 7 (only 1 to 2 from each litter) male $F_{2}$ mice from each group were decapitated. The genotypes of the experimental animals were mapped using PCR. FMR1 KO mice and WT mice were used to assess mRNA and protein levels at each time point. The left hemispheres were used for real-time PCR (4-7 mice per genotype, per age group). The right hemispheres were divided so that 3 of the right hemispheres were used for Western blot analysis and the remaining ones were used for immunohistochemistry. All experimental mice were killed by cervical dislocation and the brains were immediately removed. The hippocampi from each hemisphere were dissected out and stored at $-80^{\circ} \mathrm{C}$. All procedures used in this study adhered to the guidelines established by the Animal Ethics Committee of Zhejiang University, School of Medicine, China.

\section{Genotyping}

In the KO mice, the FMR1 gene had been disrupted by targeting a transgene to exon 5 with homologous recombination. DNA extraction was performed on tail snip tissue stored at $-80^{\circ} \mathrm{C}$. All DNA extraction procedures were performed according to the protocol of the AxyPrep Multisource Genomic DNA Miniprep Kit (Axygen Biosciences, USA). The FMR1 KO and WT mice were genotyped by PCR from DNA samples using primers provided by the Jackson Laboratory: S1, 5'-CACGAG ACTAGTGAGACGTG-3' and S2, 5'-CTTCTGGCACCTCC AGCTT-3' were used to detect the knockout allele of $400 \mathrm{bp}$, and M1, 5'-TGTGATAGAATATGCAGCATGTGA-3' and M2, 5'-CTTCTGGCACCTCCAGCTT-3' were used for the wild-type allele of $131 \mathrm{bp}$.

\section{Western blot assays}

Western blot analysis was performed on right hippocampus samples collected from FMR1 KO and WT mice ( $N=3$ per genotype, per age group). The tissue was lysed in RIPA buffer and protein concentrations were measured using the BCA Protein Assay Kit (Beyotime Institute of Biotechnology, China). FMR1 KO and WT samples containing $50 \mu \mathrm{g}$ protein were separated by $6 \%$ SDS-PAGE and electrophoretically transferred onto polyvinylidene difluoride membranes (BioRad, USA). After blocking, blots were incubated with primary antibodies recognizing $\mathrm{nNOS}$ (polyclonal antibodies against nNOS; 1:800; Santa Cruz Biotechnology, USA) and $\beta$-actin (monoclonal antibodies against $\beta$-actin; 1:3000; Beyotime Institute of Biotechnology). Blots were then incubated with secondary antibodies (goat-anti-rabbit and goat-anti-mouse, respectively; Zhongshan Institute of Biotechnology, China) in ECL reagent and exposed to autoradiographic film (Kodak).
After immunoblotting, digitized images of immunoreactive bands for target (nNOS) and control ( $\beta$-actin) proteins were imported into the Image-Pro Plus software (Media Cybernetics, USA). The ratio of nNOS to $\beta$-actin was then determined and these values were compared across development for statistical significance. Background levels were subtracted and values of $\mathrm{nNOS} / \beta$-actin at the four time points were determined to give a relative mean density for each age. The results are reported as means \pm SEM.

\section{Immunohistochemistry}

The tissue was obtained as described above. Samples were fixed in $10 \%$ neutral formalin, $\mathrm{pH} 7.4$, for $24 \mathrm{~h}$, dehydrated with distilled water for $30 \mathrm{~min}$, rinsed in $80 \%$ ethanol for $1 \mathrm{~h}$, rinsed twice in $95 \%$ ethanol for $1 \mathrm{~h}$ each, rinsed twice in $100 \%$ ethanol for $1 \mathrm{~h}$ each, rinsed twice in absolute xylene for $20 \mathrm{~min}$ each, and then embedded in paraffin at $58-60^{\circ} \mathrm{C}$ for $30 \mathrm{~min}$. After embedding, 4- $\mu \mathrm{m}$ coronal serial sections were cut (Microm HM340 Emicrotome, Microm, Germany). Two to four hippocampal sections per animal were obtained. The sections were treated with the Vision two-step strategy and high-temperature antigen retrieval. Four-micron thick paraffinembedded sections were deparaffinized twice, 10 min each in $100 \%$ xylene, and then hydrated with two 5-min rinses in $100 \%$ ethanol, two 3-min rinses in 95\% ethanol, two 3-min rinses in $80 \%$ ethanol, and two $3-$ min rinses in $70 \%$ ethanol. The sections were then rinsed in distilled water and the slides were placed in a pressure cooker filled with boiling sodium citrate buffer, pH 6.0, and heated under pressure. One minute and forty seconds after steaming, the pressure cooker was removed from the heating source and cooled down to room temperature with tap water. The container was opened, the slides were rinsed 3 times for 5 min each with phosphate-buffered saline (PBS), incubated with $3 \%$ hydrogen peroxide for $10 \mathrm{~min}$, and then rinsed three times in PBS for 5 min each. The slides were then incubated with the same primary antibody as used for the Western blot (1:60) for $1 \mathrm{~h}$ in a humid chamber at $37^{\circ} \mathrm{C}$. After three 5-min rinses in PBS, the slides were incubated with antirabbit HRP-conjugated secondary antibody (1:30, Zhongshan Institute of Biotechnology) for $30 \mathrm{~min}$ at $37^{\circ} \mathrm{C}$. The sections were rinsed again in PBS 3 times, 5-min each and staining was then visualized using diaminobenzidine as the chromogen. The slides were observed and examined for color change under a light microscope (Olympus BX60, Japan) in order to determine when to terminate chromogen development. Antibody staining was followed by a counterstain with hematoxylin for $1 \mathrm{~min}$. For antibody specificity controls, the primary antibody was omitted from the sections and substituted with PBS as a negative control. Two single-blind pathologists independently assessed and reported the immunohistochemical staining. Representative images were taken of one of the hippocampi stained per genotype, per age.

\section{cDNA preparation}

Total RNA from the left hippocampus of each animal was 
isolated using the AxyPrep Multisource Total RNAMiniprep Kit according to manufacturer instructions (Axygen Biosciences). The yield of total RNA was quantified spectrophotometrically by measuring the absorbance at 260 and $280 \mathrm{~nm}$ and purity was confirmed by the $A_{260} / A_{280}$ ratio (range: $1.8 \pm 0.2$ ). Two micrograms of total RNA from each sample was reverse transcribed into cDNA using the SYBR PrimeScript ${ }^{\mathrm{TM}}$ RT-PCR kit (TaKaRa Code: DRR063A) according to manufacturer instructions (Takara Bio Inc., Japan).

\section{Real-time PCR}

Semi-quantitative real-time PCR was used to examine the expression of nNOS messenger RNA in the left hippocampi using total RNA. cDNAs from reversed transcribed RNA were amplified and analyzed on a Thermal Cycler 7500 Real-Time PCR System (Applied Biosystems, USA) using an SYBR PrimeScript ${ }^{\mathrm{TM}}$ PCR Kit (Takara Bio Inc.). Two steps of realtime PCR amplification were run: hot start for $3 \mathrm{~min}$ at $94^{\circ} \mathrm{C}$, denaturation for $15 \mathrm{~s}$ at $94^{\circ} \mathrm{C}$, and annealing for $1 \mathrm{~min}$ at $62^{\circ} \mathrm{C}$ for 40 cycles. Melt curve analyses verified the formation of a single-desired PCR product. Mouse $\beta$-actin was used as an internal control, and self-designed primer sequences were used, 5'-GTGGCCATCGTGTCCTACCATAC-3' and 5'-GTTTCG AGGCAGGTGGAAGCTA-3' for the detection of nNOS, 5'-CCGTTTCTCCTGGCTCAGTTTA-3' and 5'-CCCCAATACC ACATCATCCAT-3' for the detection of $\beta$-actin. The amount of target transcript, normalized by an endogenous reference, was calculated as $2^{-\Delta C T}$, where $\Delta C T$ are the differences in threshold cycles for the target (nNOS) and reference ( $\beta$-actin) measured in the samples. The level of the endogenous reference gene is constantly expressed, thus $2^{-\Delta C T}$ as relative fold-change over the values of the endogenous reference represents the target gene mRNA levels. The specificity of each reaction was controlled by melting curve analysis. A negative PCR control containing water instead of cDNA was performed. Forty-seven FMR1 KO and 47 WT mice were analyzed at each time point. The results are reported as means \pm SEM.

\section{Statistical analysis}

Statistical analysis was conducted using the SPSS software (10.0) and data are reported as means \pm SEM. The Student $t$ test was used for time-matched group comparisons. Atwo-tailed $P$ value of less than 0.05 was considered to be significant.

\section{Results}

\section{Expression of nNOS protein}

Immunohistochemical staining detected a wide distribution of nNOS in developing hippocampi of FMR1 KO and WT mice. Positive staining was observed in all developing FMR1 KO and WT mice, localizing diffusely as well as to the neuronal cytoplasm (Figure 1). Among all the time points, the most robust overall immunoreactivity in both genotypes was observed in hippocampi of P7 mice (Figure 1). However, in the FMR1 KO group nNOS immunoreactivity intensity particularly in neuronal cell bodies decreased at P14 and P21 and then increased at P28 (see Figure 1B, D, F, and $H)$. There was no obvious decrease in neuronal perikaryon staining intensity in the WT group at P14 and P21 (see Fig-
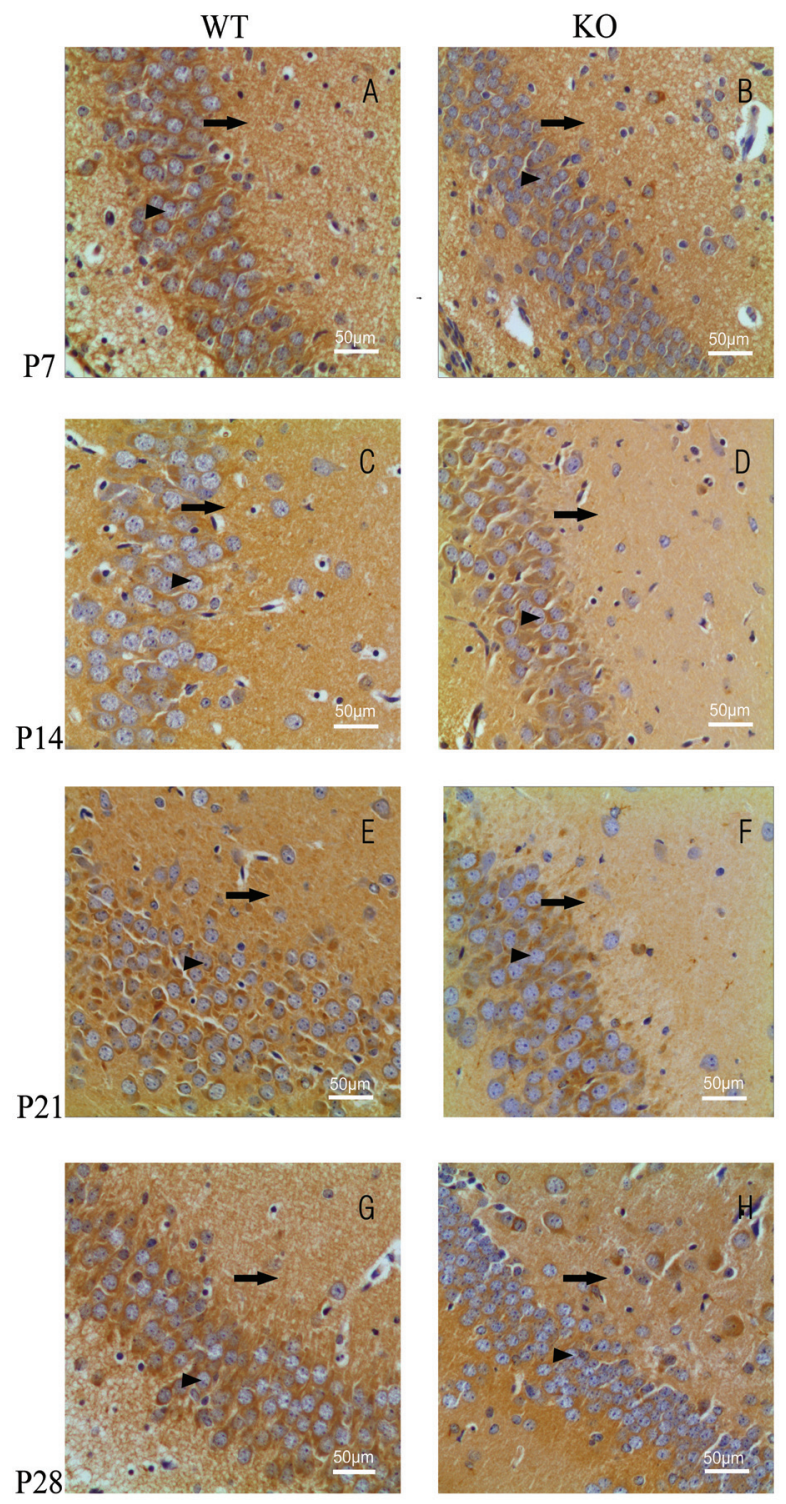

Figure 1. Immunocytochemical detection of neuronal nitric oxide synthase (nNOS) in hippocampi of WT and FMR1 KO mice at P7, P14, P21, and P28 ( $=1)$. nNOS immunoreactivity was visualized using diaminobenzidine as brown cytoplasmic stain (arrows), whereas nuclear immunoreactivity was visualized using hematoxylin as a blue stain (arrowheads). Representative images from the Cornu ammonis area 1 region of WT (Panels A, C, E, G) and KO (Panels B, D, F, H) pairs are shown in an age-matched fashion (magnification $=200$; scale bar $=50 \mu \mathrm{m}$ ). $\mathrm{WT}=$ wild-type mice; FMR1 $\mathrm{KO}=$ fragile $\mathrm{X}$ mental retardation 1 gene knockout mice; P7, P14, P21, P28 = postnatal days 7, 14, 21 , and 28 , respectively. 
ure $1 A, C, E$, and $G$ ). In order to substantiate the qualitative immunostaining findings, protein levels of hippocampal nNOS were examined at each time point by Western blot analysis. In agreement with the immunohistochemical findings, the level of nNOS protein in the FMR1 KO group on P21 was significantly lower than that in the WT group $(\mathrm{KO}=0.3015 \pm 0.0897, \mathrm{WT}=1.7542 \pm 0.5455 ; \mathrm{P}$ $<0.05$; Figure 2). The temporal profile of nNOS protein levels in the WT group demonstrated a decrease in protein levels at P14, followed by a significant increase by P21 that was maintained at P28 (Figure 2). FMR1 KO mice also presented a decrease in nNOS protein levels at P14. However, protein levels remained low at P21 and were significantly lower than those of WT mice at this age (Figure 2). The nNOS protein levels in FMR1 $\mathrm{KO}$ animals significantly increased to WT values by P28 (Figure 2). These data support the immunohistochemical findings of temporal differences in nNOS protein levels in the hippocampi of $\mathrm{KO}$ and WT mice. More importantly, they indicate that $\mathrm{KO}$ mice may present a temporal delay at P21 in a required increase in nNOS protein levels in the hippocampus.

\section{Expression of nNOS mRNA}

In order to determine whether the changes in nNOS protein levels might be due to transcriptional regulatory differences, $\mathrm{nNOS}$ mRNA levels were compared at each age (P7, P14, P21, and P28) for each genotype. The profiles of the trends of nNOS mRNA expression changes in the FMR1 $\mathrm{KO}$ and WT groups were similar. The levels of nNOS mRNA were lower at P7, significantly increased at P14, significantly decreased at P21, and remained at those levels on P28 (Figure 3). However, at P21 the FMR1 KO mice showed significantly less mRNA compared to WT mice (KO $=0.2857 \pm 0.0150, \mathrm{WT}=0.5646 \pm 0.0657 ; \mathrm{P}<$ 0.05 ; Figure 3). This significant reduction in nNOS mRNA levels at P21 corresponded precisely with the significantly lower levels of nNOS protein in FMR1 KO mice at this age (Figure 2).

\section{Discussion}

Current thought regarding spine morphogenesis assumes that spines begin as filopodia, proceed to thin or stubby spines and ending as mushroom spines (12). However, the neuropathology associated with FXS exhibits significantly longer dendritic spines and fewer shorter spines, as well as more spines with immature appearing morphology and fewer with mature appearing morphology. This has been demonstrated in both FXS brains $(3,13,14)$

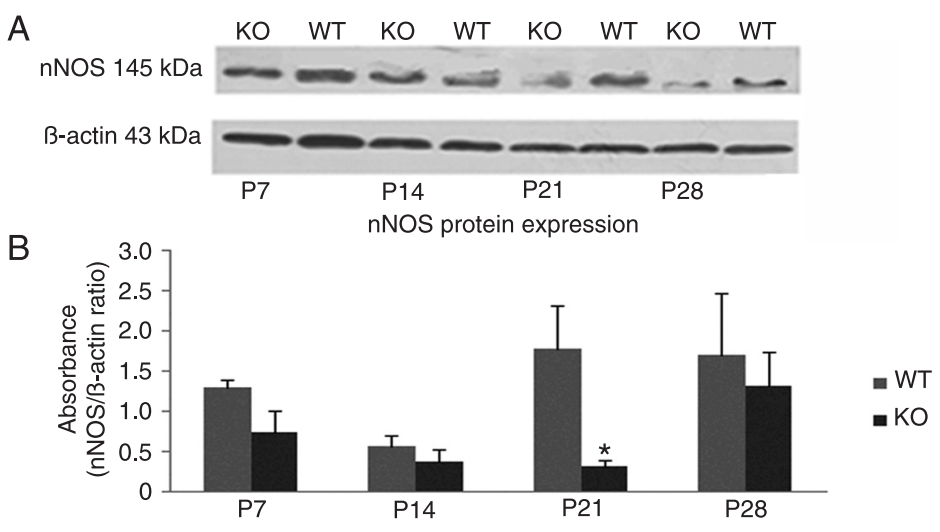

Figure 2. Western blot detection of neuronal nitric oxide synthase (nNOS) in hippocampi of WT and FMR1 KO mice at P7, P14, P21, and P28 ( $=3$ per genotype, per age group). $A$, Representative blots of nNOS protein levels at each age in the hippocampi of the FMR1 KO and WT groups were compared to $\beta$-actin levels (loading control). $B$, Absorbance of nNOS immunoreactive bands normalized to their respective $\beta$-actin loading control from 3 animals per genotype per age group were averaged and are reported as means \pm SEM. ${ }^{*} \mathrm{P}<0.05$ vs WT on P21 (two-tailed Student $t$-test). WT = wild-type mice; FMR1 KO = fragile $X$ mental retardation 1 gene knockout mice; $P 7$, P14, P21, P28 = postnatal days $7,14,21$, and 28, respectively.

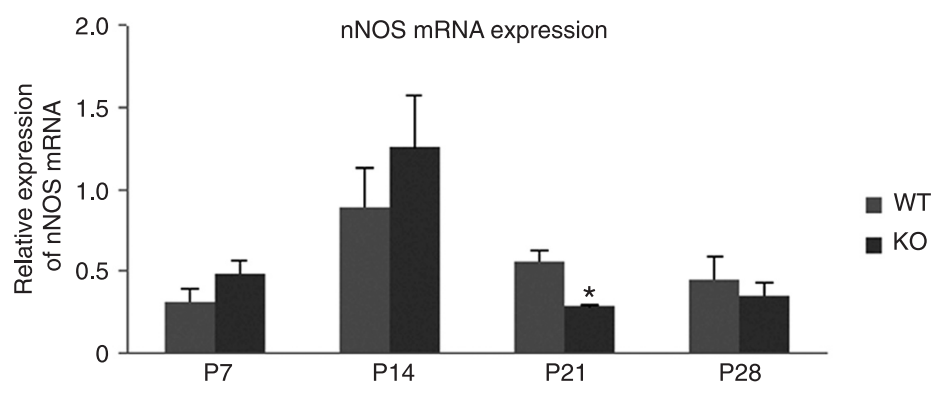

Figure 3. Comparison of neuronal nitric oxide synthase (nNOS) mRNA in hippocampi of WT and FMR1 KO mice by RT-PCR at P7, P14, P21, and P28 ( $N=4-7$ mice per genotype, per age group). $\Delta \mathrm{Ct}=\mathrm{Ct}(\mathrm{nNOS})-\mathrm{Ct}$ ( $\beta$-actin), where $\mathrm{Ct}=$ cycle threshold, $\beta$-actin is the endogenous reference. Relative nNOS mRNA expression: $\mathrm{P} \Delta \mathrm{Ct}=2^{-\Delta \mathrm{Ct}}$, representing nNOS mRNA levels. The final data in the graph were magnified to $1000 \times 2^{-\Delta C t}$. Data are reported as means \pm SEM. ${ }^{*} \mathrm{P}<0.05$ vs WT on P21 (two-tailed Student $t$ test). $\mathrm{mRNA}=$ messenger ribonucleic acid; $\mathrm{WT}$ = wild-type mice; FMR1 KO = fragile $\mathrm{X}$ mental retardation 1 gene knockout mice; P7, P14, P21, P28 = postnatal days $7,14,21$, and 28 , respectively. and in brains of FMR1 KO mice, an animal model of FXS $(4,15)$.

In the first to second postnatal week, numerous dendritic filopodia extend to form synaptic contacts with axons (16). At P14, filopodia shrink and more spines are formed (5). At P21, most filopodia complete a functional and morphological transition to spines, suggesting that P21 is a critical period for spinogenesis and synaptogenesis (11,17-19). Subsequent to this, synaptic maturation and stabilization proceed on until P28 (5). 
The results presented here demonstrate that the protein levels of nNOS in WT mice were relatively high at P7 when dendritic filopodia are abundant, decreased slightly at P14, dramatically increased to a peak at P21 when filopodia gradually complete spine transition, and no additional change occurred at P28 compared to P21 when spines proceed to further stabilization. The protein levels of FMR1 KO mice were significantly decreased particularly at P21 compared to WT mice and then increased to levels comparable to those of WT animals by P28. This temporal delay in the expression of nNOS at the critical period of filopodia to spine transition is likely to cause retardation of normal spinogenesis and synaptogenesis development in the hippocampus.

The mRNA expression profile of WT mice demonstrated a significant peak of nNOS mRNA expression at P14 compared to all other time points. One possibility for this increase in mRNA at this age may be that nNOS mRNA levels were up-regulated ahead of the increase in protein levels observed at P21 to facilitate a maximal increase in protein levels, thus leading to an increase in NO during this critical period of filopodia to spine transition. Compared to the WT group, the nNOS mRNA changes in FMR1 KO mice demonstrated the same trend with the peak of $\mathrm{mRNA}$ levels at P14, but levels of mRNA at P21 were significantly less than WT controls. Therefore, both protein and mRNA profiles in the FMR1 KO animals demonstrated a significant reduction at P21. This deficiency probably results in an inadequate production of $\mathrm{NO}$ during the critical period for synapse and spine maturation. Although we have not measured NO levels per se, we suggest that the attenuated nNOS expression probably results in down-regulation of $\mathrm{NO}$ production in FMR1 KO mice. Thus, we speculate that NO

\section{References}

1. Brouwer JR, Willemsen R, Oostra BA. The FMR1 gene and fragile X-associated tremor/ataxia syndrome. Am J Med Genet B Neuropsychiatr Genet 2009; 150B: 782-798.

2. Penagarikano O, Mulle JG, Warren ST. The pathophysiology of fragile x syndrome. Annu Rev Genomics Hum Genet 2007; 8: 109-129.

3. Grossman AW, Elisseou NM, McKinney BC, Greenough WT. Hippocampal pyramidal cells in adult Fmr1 knockout mice exhibit an immature-appearing profile of dendritic spines. Brain Res 2006; 1084: 158-164.

4. Nimchinsky EA, Oberlander AM, Svoboda K. Abnormal development of dendritic spines in FMR1 knock-out mice. $J$ Neurosci 2001; 21: 5139-5146.

5. Matsuno H, Okabe S, Mishina M, Yanagida T, Mori K, Yoshihara Y. Telencephalin slows spine maturation. J Neurosci 2006; 26: 1776-1786

6. Morita A, Yamashita N, Sasaki Y, Uchida Y, Nakajima O, Nakamura $F$, et al. Regulation of dendritic branching and spine maturation by semaphorin3A-Fyn signaling. J Neurosci 2006; 26: 2971-2980. deficiency during spinogenesis and synaptogenesis may retard dendritic spine maturation in FXS.

There is evidence supporting the notion that NO production is indeed required for spine maturation. For example, $\mathrm{NO}$ as a messenger/modulator in synaptogenesis can stimulate axons located around the spines to differentiate into varicosities and then form multi-innervated spines (9). This mechanism is mediated by the targeting and mobilization of synaptic vesicles in the presynaptic cell, enhancing transmitter release and then activating protein kinase signaling cascades in the postsynaptic cell $(20,21)$. In addition, NO can promote transcription and translation, which are critical for long-term synaptic plasticity and memory formation (2224). As we know, long-term potentiation (LTP) can increase synaptogenesis and enhance connectivity between neurons to facilitate memory storage. Hippocampal LTP triggered by $\mathrm{NO}$ is a process known to be involved in mammalian learning and memory in many studies (25-31). The morphological consequences of hippocampal LTP include enlarged spine heads, shortened spine necks, formation of perforated synapses, bifurcation of spines, and even formation of new spines in the vicinity of the activated spines $(32,33)$. Moreover, NOS inhibition during synaptic maturation decreases synapsin I immunoreactivity in rat hippocampi, indicating a reduction in synaptic density (10).

These findings suggest that it is plausible to infer from our study that impaired NO production may be one of the reasons for spine retardation in FXS.

\section{Acknowledgments}

We thank Chaochun Zou (Hangzhou, Zhejiang, China) for technical assistance.
7. Centonze D, Siracusano A, Calabresi P, Bernardi G. The Project for a Scientific Psychology (1895): a Freudian anticipation of LTP-memory connection theory. Brain Res Brain Res Rev 2004; 46: 310-314.

8. Nikonenko I, Boda B, Steen S, Knott G, Welker E, Muller D. PSD-95 promotes synaptogenesis and multiinnervated spine formation through nitric oxide signaling. J Cell Biol 2008; 183: 1115-1127.

9. Nikonenko I, Jourdain P, Muller D. Presynaptic remodeling contributes to activity-dependent synaptogenesis. J Neurosci 2003; 23: 8498-8505.

10. Sanchez-Islas E, Leon-Olea M. Nitric oxide synthase inhibition during synaptic maturation decreases synapsin I immunoreactivity in rat brain. Nitric Oxide 2004; 10: 141-149.

11. Grabrucker A, Vaida B, Bockmann J, Boeckers TM. Synaptogenesis of hippocampal neurons in primary cell culture. Cell Tissue Res 2009; 338: 333-341.

12. Yuste R, Bonhoeffer T. Genesis of dendritic spines: insights from ultrastructural and imaging studies. Nat Rev Neurosci 2004; 5: 24-34. 
13. Irwin SA, Patel B, Idupulapati M, Harris JB, Crisostomo RA, Larsen BP, et al. Abnormal dendritic spine characteristics in the temporal and visual cortices of patients with fragile-X syndrome: a quantitative examination. Am J Med Genet 2001; 98: 161-167.

14. Wisniewski KE, Segan SM, Miezejeski CM, Sersen EA, Rudelli RD. The Fra(X) syndrome: neurological, electrophysiological, and neuropathological abnormalities. Am J Med Genet 1991; 38: 476-480.

15. Galvez R, Greenough WT. Sequence of abnormal dendritic spine development in primary somatosensory cortex of a mouse model of the fragile $X$ mental retardation syndrome. Am J Med Genet A 2005; 135: 155-160.

16. Fiala JC, Feinberg M, Popov V, Harris KM. Synaptogenesis via dendritic filopodia in developing hippocampal area CA1. J Neurosci 1998; 18: 8900-8911.

17. Grutzendler J, Kasthuri N, Gan WB. Long-term dendritic spine stability in the adult cortex. Nature 2002; 420: 812816.

18. McCroskery S, Bailey A, Lin L, Daniels MP. Transmembrane agrin regulates dendritic filopodia and synapse formation in mature hippocampal neuron cultures. Neuroscience 2009; 163: 168-179.

19. Papa M, Bundman MC, Greenberger V, Segal M. Morphological analysis of dendritic spine development in primary cultures of hippocampal neurons. J Neurosci 1995; 15 : $1-11$.

20. Hawkins RD, Kandel ER, Siegelbaum SA. Learning to modulate transmitter release: themes and variations in synaptic plasticity. Annu Rev Neurosci 1993; 16: 625-665.

21. Hawkins RD, Son $H$, Arancio $O$. Nitric oxide as a retrograde messenger during long-term potentiation in hippocampus. Prog Brain Res 1998; 118: 155-172.

22. Chien WL, Liang KC, Teng CM, Kuo SC, Lee FY, Fu WM. Enhancement of long-term potentiation by a potent nitric oxide-guanylyl cyclase activator, 3-(5-hydroxymethyl-2furyl)-1-benzyl-indazole. Mol Pharmacol 2003; 63: 1322-
1328.

23. Ota KT, Monsey MS, Wu MS, Young GJ, Schafe GE. Synaptic plasticity and NO-cGMP-PKG signaling coordinately regulate ERK-driven gene expression in the lateral amygdala and in the auditory thalamus following Pavlovian fear conditioning. Learn Mem 2010; 17: 221-235.

24. Taqatqeh F, Mergia E, Neitz A, Eysel UT, Koesling D, Mittmann $\mathrm{T}$. More than a retrograde messenger: nitric oxide needs two cGMP pathways to induce hippocampal long-term potentiation. J Neurosci 2009; 29: 9344-9350.

25. Arancio O, Kiebler M, Lee CJ, Lev-Ram V, Tsien RY, Kandel $E R$, et al. Nitric oxide acts directly in the presynaptic neuron to produce long-term potentiation in cultured hippocampal neurons. Cell 1996; 87: 1025-1035.

26. Bon CL, Garthwaite J. On the role of nitric oxide in hippocampal long-term potentiation. J Neurosci 2003; 23: 1941-1948.

27. Garthwaite J, Boulton CL. Nitric oxide signaling in the central nervous system. Annu Rev Physiol 1995; 57: 683-706.

28. Mutlu O, Ulak G, Belzung C. Effects of nitric oxide synthase inhibitors 1-(2-trifluoromethylphenyl)-imidazole (TRIM) and 7-nitroindazole (7-NI) on learning and memory in mice. Fundam Clin Pharmacol 2011; 25: 368-377.

29. Schuman EM, Madison DV. Nitric oxide and synaptic function. Annu Rev Neurosci 1994; 17: 153-183.

30. Schuman EM, Madison DV. A requirement for the intercellular messenger nitric oxide in long-term potentiation. Science 1991; 254: 1503-1506.

31. Schuman EM, Madison DV. Locally distributed synaptic potentiation in the hippocampus. Science 1994; 263: 532536.

32. Yuste R, Bonhoeffer T. Morphological changes in dendritic spines associated with long-term synaptic plasticity. Annu Rev Neurosci 2001; 24: 1071-1089.

33. Harris KM, Fiala JC, Ostroff L. Structural changes at dendritic spine synapses during long-term potentiation. Philos Trans R Soc Lond B Biol Sci 2003; 358: 745-748. 